\title{
A New Method to Fabricate 3D Electron Tomography Sample Using FIB Technique
}

Xiongyao Wang, Ross Lockwood, Doug Vick, Al Meldrum, Marek Malac,

National Institute for Nanotechnology, Edmonton, 11421 Saskatchewan Drive, and Department of Physics, University of Alberta, Edmonton, Canada

Electron tomography (ET) in a TEM has been widely used to characterize materials at sub-10 $\mathrm{nm}$ spatial resolution. The first example of tomographic imaging in an electron microscope was reported four decades ago [1,2].However, due to the fast development of processing capacity of modern computers it is only in the past few years that ET in (scanning) transmission electron microscopy ((S)TEM) has become a more viable option for investigating the morphologies, spatial distribution and chemical compositions of nanostructures [3].

There are many factors which can affect the quality of the final reconstructed results [3] such as reconstructed algorithms, the experimental conditions of recording the projected images. In practice, the increasing thickness of the projected sample or the shielding by the holder and grid bars leading to missing range of angles in the collected data is an important experimental factor limiting the quality of the reconstructed results. Although double-axis tilt electron tomography was proposed to overcome the problem, the alignment appears to be complicated. A feasible way to overcome the problem is to fabricate a rod-shaped sample and employ the $\pm 90^{\circ}$ tilt holder [4].Commercial $\pm 90^{\circ}$ tilt holder right now is available [5]. However, decreasing the thickness of the sample is not always simple. Here we report a convenient method for sample preparation suitable for high tilt ET from materials that are thin films to begin with.

The method combines deposition of the sample onto thin film substrate, such as amorphous carbon or SiN membrane, followed by focused ion beam (FIB) fabrication. Depending on the substrate used the sample can be tilted to very high angles, typically $\pm 75^{\circ}$, while eliminating the need for needle shaped sample for $\pm 90^{\circ}$ tilt. First, the sample is directly prepared as thin film or deposited onto a thin film support film. FIB is then used to cut out two large rectangles of the thin film leaving a thin bar of the original film for ET experiment as shown in an SEM image in Fig 1. The bar was aligned to be parallel to the side of the grid bar for easy alignment of the bar long axis parallel to the tilt axis.

Here we give an example of $\mathrm{SiO}_{1.5}$ with Erbium thin film sample. The sample was deposited onto Mica substrate by simultaneous thermal evaporation of silicon monoxide ( $\mathrm{SiO}$ ), silica $\left(\mathrm{SiO}_{2}\right)$, and metallic erbium $(\mathrm{Er})$. The film composition of $\mathrm{SiO}_{1.5}(10$ at.\% excess $\mathrm{Si})$ with an erbium doping level of about $6 \times 10^{20} \mathrm{~cm}^{-3}$ (1 at.\%), and a film thickness of $20 \mathrm{~nm}$. The $\mathrm{SiO}_{1.5}$ with Er doped thin film was floated from Mica substrate on distilled water and collected onto molybdenum TEM grid. Subsequent annealing in forming gas at $1000^{\circ} \mathrm{C}$ results in the $\mathrm{Si}$ and $\mathrm{SiO}_{2}$ phases segregation and growth of silicon nanocrystals and also promotes erbium clustering.

We analysed the sample in a JEOL 2200 FS TEM/STEM equipped with an Omega filter allowing us to image both Er and Si nanoclusters in $\mathrm{SiO}_{1.5}$ : Er films simultaneously [6]. Fig 2 
shows one recorded image of Si nanocluster from EFTEM and Er from HAADF image in STEM mode. A series of tilt images was attained. The $3 \mathrm{D}$ reconstruction is in the process.

The method takes advantage of deposition technique to control the thickness of the sample. Although the method cannot cover the full $\pm 90^{\circ}$ tilt range, it can reach $\pm 75^{\circ}$ tilt range which is sufficient in many practical applications [5]. The precondition is that the side of the cut rectangular perpendicular to the bar is four times length of the width of the bar, which is easy to be realized. It appears that the reported method simplifies the fabricating process of the sample in FIB. The process also perhaps also reduces Ga contamination compare to needle shape sample method since only two sides of a thin film are FIB milled as opposed to extensive milling of entire needle height in standard needle preparation method. Conceivably, the method can be also used for samples deposited onto thin substrate from solution and perhaps the substrate can be milled before the sample is deposited onto it. The fact that there is large area in addition the narrow bridge for tomography provides ample opportunity for additional analysis in a TEM.

[1] De Rosier,D.J., Klug,A.. Reconstruction of three dimensional structures from electron micrographs. Nature 217,130-134 (1968).

[2] Hart, R.G.. Electron microscopy of unstained biological material: the polytropic montage. Science 159, 14641467 (1968).

[3] J. Frank, Three-Dimensional Electron Microscopy of Macromolecular Assemblies, Academic Press, San Diego, 1996.

[4] H. Jinnai, R.J. Spontak, Transmission electron microtomography in polymer research. Polymer. 50 (2009) 1067.

[5] Paul A. Midgley and Rafal E. Dunin-Borkowski. Electron tomography and holography in materials science.

Nature Mater. 8, 271-280 (2009).

[6] P. Li , X. Wang, M. Malac, R. Egerton, A. Meldrum, X. Liang, F. Lenz, J. Wang Microscopy \& Microanalysis, Vol 15, Suppl.2, p.1256, (2009).

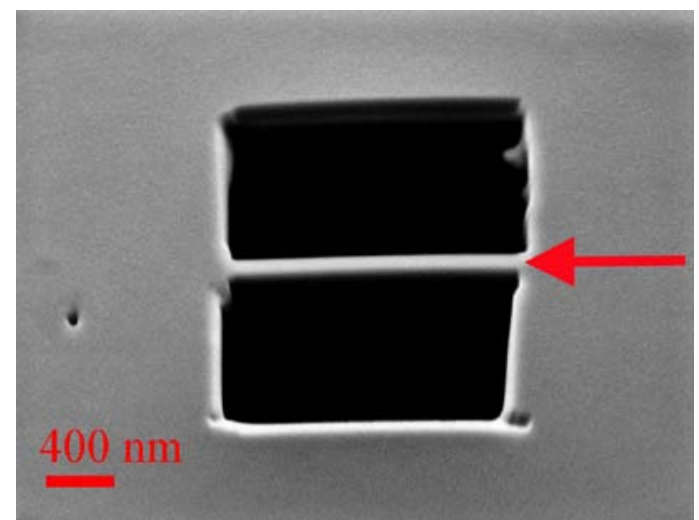

Fig 1 the bar sample prepared in FIB. The rotating axis along the direction of the red arrow.

The narrow bridge marked by the arrow is examined by electron tomography. Plenty sample area is available for additional TEM examination outside the narrow bridge.

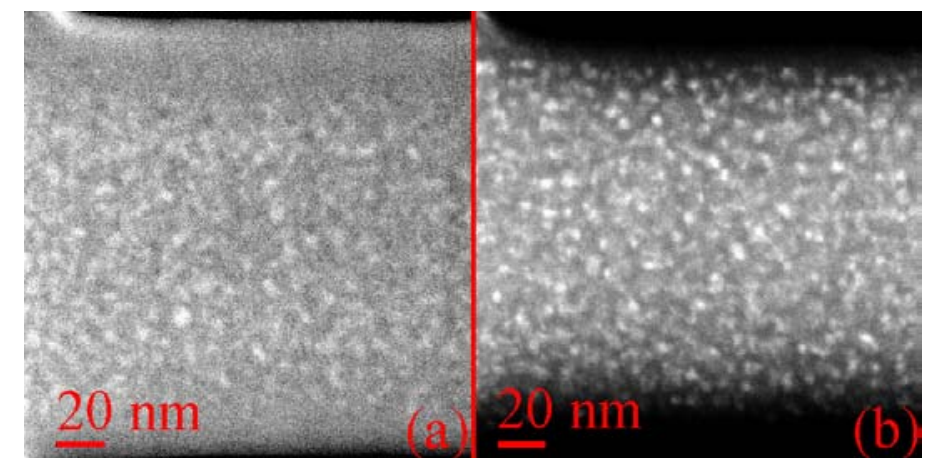

Fig 2 a) Si nanoclusters visualized using EFTEM and b) Er clusters visualized using HAADF. Data were collected simultaneously from the same area in STEM mode. 